\title{
BEM-ESTAR DE PEIXES E A QUESTÃO DA SENCIÊNCIA
}

\section{(Fish welfare and the problem of sentience)}

\section{PEDRAZZANI, A.S. ${ }^{\text {; }}$ FERNANDES-DE-CASTILHO, M. ${ }^{2}$; CARNEIRO, P.C.F.3; MOLENTO, C. F. M. ${ }^{1}$}

1 LABEA-UFPR - Laboratório de Bem-estar Animal, Universidade Federal do Paraná

2 Research Center on Animal Welfare - RECAW, Departamento de Fisiologia, Universidade Federal do Paraná.

3 Embrapa Tabuleiros Costeiros, Aracaju, SE.

\begin{abstract}
RESUMO - No Brasil, assim como em outras partes do mundo, a preocupação com o bem-estar dos peixes encontra-se em seus passos iniciais. O bem-estar de peixes, se comparado com o bemestar de outras espécies de animais de produção, é um tópico praticamente ausente para consumidores, produtores e legislação vigente. Porém, as evidências anatômicas, fisiológicas, comportamentais, evolutivas e farmacológicas sugerem que os peixes experimentam sentimentos como dor, medo e outros de maneira similar aos demais vertebrados. Isso sugere que os peixes têm potencialmente a capacidade de sofrer e apresentar outros sentimentos, ou seja, que os peixes são seres sencientes. Esta revisão teve como objetivo discutir aspectos referentes à dor e à presença de consciência nos peixes, visando colaborar com o entendimento da senciência nesses animais. Uma vez reconhecida a senciência em peixes, estes animais são incluídos nas considerações éticas acerca de não serem submetidos a sofrimento.
\end{abstract}

Palavras-chave: Senciência; cognição; consciência; dor; teleósteo.
ABSTRACT - In Brazil, as well as in other parts of the World, the concern about fish welfare is in its initials steps. Fish welfare, if compared to the welfare of other species of farm animals, is practically an absent topic for consumers, producers and current law. However, anatomic, physiologic, behavioral, evolutive and pharmacologic evidences suggest that fish experience feelings such as pain, fear and others, in ways that are similar to other vertebrates. This suggests that fish are potentially capable of experiencing suffering and other feelings; in other words, that fish are sentient. The objective of this review was to discuss pain and consciousness in fish, in order to collaborate with sentience understanding in these animals. Once fishes are recognized as sentient beings, these animals are then included into ethical considerations regarding the avoidance of suffering infliction.

Key-words: Sentience; cognition; consciousness; pain; teleost.

1 E-mail: anasilviap@yahoo.com.br 


\section{INTRODUÇÃO}

Os peixes são amplamente utilizados em áreas distintas, seja em pesca e aqüicultura, na investigação científica, ou ainda como animais ornamentais. A preocupação com o bem-estar dos peixes durante os processos produtivos é incipiente se comparada com aquela de outras espécies de animais de produção, sendo ainda um tópico praticamente ausente para consumidores, produtores e legislação vigente. Isto é comprovado pela ausência de capítulos sobre peixes em livros-texto importantes na área de bem-estar animal (ROLLIN, 1995; FRASER e BROOM, 1996; BENSON e ROLLIN, 2004; VAARST et al., 2004). Entretanto, recentemente este cenário vem se alterando, com o aumento de publicações internacionais, de relatórios e livros dedicados ao bem-estar de peixes (LYMBERY, 2002; ERICKSON, 2003; BRANSON, 2007; PEDRAZZANI et al., 2007). Adicionalmente, nos últimos sete anos foram publicados nove artigos importantes sobre o referido assunto no periódico científico Disease of Aquatic Organisms, seis artigos no Fish and Fisheries, doze no Aquaculture Research, dezoito no Journal of Fish Biology e trinta e cinco artigos no Applied Animal Behaviour Science.

A partir de conceitos de bem-estar (APPLEBY, 1999; BROOM e MOLENTO, 2004; DUNCAN, 2005; KIRKWOOD, 2006), pode-se inferir que o potencial de senciência seja um pré-requisito para a consideração de bem-estar, pois a senciência está relacionada à capacidade de sentir. Para DUNCAN e PETHERICK (1991), não é necessariamente o estado de saúde ou a quantidade de estresse de um animal que importa para seu bem-estar, mas a posse e o estado de um número de capacidades cognitivas que formam a base da senciência, ou seja, o bem-estar animal é dependente unicamente das necessidades cognitivas. Em geral, se as necessidades cognitivas são atendidas, estas irão proteger as necessidades físicas dos animais. As necessidades cognitivas incluem os sentimentos e os meios pelos quais os animais aprendem, respondem aos estímulos, e antecipam eventos futuros (DOLAN, 1999). O sentimento envolve "sentir e perceber" o que ocorre com o organismo, ou seja, um animal estaria consciente de um estímulo se ele o sentisse (DUNCAN e PETHERICK, 1991). Para se trabalhar com o bem-estar de peixes, em primeira instância deve-se buscar o entendimento de habilidades como sentir dor, frio, conforto, desconforto, e de diferenciar conscientemente os estados internos como "bom ou ruim", "prazeroso ou desagradável" (VOLPATO et al., 2007). Ou seja, para se levar em consideração o bem-estar dos peixes, estes devem demonstrar características cognitivas compatíveis com aquelas de seres sencientes.
Esta revisão tem como objetivo discutir aspectos referentes à cognição e à presença de consciência nos peixes, visando colaborar com o entendimento da senciência nesses animais. Adicionalmente, são discutidos aspectos anatomo-fisiológicos relacionados à nocicepção e à percepção da dor, ao sistema límbico e ao sistema dopaminérgico dos peixes teleósteos.

\section{COGNIÇÃO E CONSCIÊNCIA}

Um modo simples de caracterizar a cognição está relacionado com a capacidade do indivíduo de reconhecer os estímulos internos e externos (DUNCAN, 1996). De acordo com SHETTLEWORTH (2001), a cognição envolve todos os processos mentais que os animais utilizam para adquirir informações sobre seu ambiente, para armazená-las, evocá-las, e para a consideração dessas informações em suas decisões.

A consciência pode ser definida como a habilidade de gerar uma cena mental, na qual diversas informações são integradas com o propósito de direcionar o comportamento (EDELMAN e TONONI, 2000). Para GRIFFIN (1992), consciência é o que o animal percebe num dado momento a respeito de sua situação imediata. Um animal experimenta níveis simples de consciência acerca de objetos e eventos, prestando atenção a imagens mentais internas ou representações de objetos e eventos. Estas representações podem ser situações com as quais o animal se defronta no presente, lembranças, ou antecipações de situações futuras. Isso induz o animal a efetuar comparações entre duas ou mais representações e decidir sobre o comportamento que acredita ser capaz de levar a resultados desejados, ou mesmo de evitar resultados negativos. Portanto, a consciência é considerada como uma forma de cognição mais complexa e elevada (GRIFFIN e SPECK, 2004).

Embora existam questionamentos com relação à consideração dos animais como seres conscientes, a tendência atual no âmbito científico é para o reconhecimento de tal capacidade. Primeiramente, a pesquisa de estruturas neuronais correspondentes de consciência não encontrou nenhuma estrutura anatômica de produção de consciência que seja limitada ao cérebro humano (PRADA, 2000; BSHARY et al., 2002; CHANDROO et al., 2004; GRIFFIN e SPECK, 2004; KIRKWOOD, 2006). Em segundo lugar, respostas apropriadas a novos desafios aos quais os animais não estão programados geneticamente, ou por experiências prévias, são sugestivas de consciência animal: tamanha versatilidade provavelmente é organizada pelo pensamento 
consciente. Ainda, a comunicação animal freqüentemente revela evidência de experiências subjetivas (GRIFFIN e SPECK, 2004; BEKOFF, 2006).

Os peixes estiveram pouco presentes na pesquisa em cognição até o final do século passado, quando a maior parte da literatura de peixes era descritiva. Provavelmente isso tenha se dado devido à distância filogenética entre os mamíferos e os peixes, considerando assim o comportamento sob aspecto mecanicista e as reações de forma inconsciente. Porém, BSHARY et al. (2002) classificaram fenômenos comportamentais observados em peixes em duas categorias, conforme a hipótese de inteligência de Machiavel, que afirma que o complexo social causa o alargamento do neocórtex: inteligência social e inteligência ambiental. Os fenômenos classificados como inteligência social têm como exemplos comportamentos em grupos individualizados e estratégias sociais correspondentes, aprendizado social e, pela primeira vez, apresentaram exemplos de caça cooperativa entre a moréia gigante (Gymnothorax javanicus) e a garoupa de coral (Plectopomus pessuliferus). Adicionalmente, foram discutidos vários fenômenos achados em simbiose de limpeza que indicam que os peixes-limpadores são particularmente apropriados para se testar a "hipótese de inteligência de Machiavel” (BSHARY et al., 2002). Já os fenômenos classificados como inteligência ambiental foram exemplos de habilidades especiais para alimentação, o uso de substratos como ferramentas, memória espacial e memória em longo prazo. Isto demonstra que os peixes apresentaram soluções para quase todos os problemas que supostamente levaram à evolução para o aumento do neocórtex e o surgimento de habilidades cognitivas em primatas.

A investigação efetuada em diversas espécies revelou comportamentos indicadores de representações mentais declarativas e de uma memória e capacidade de aprendizagem complexas e flexíveis (GALHARDO e OLIVEIRA, 2006). A TABELA 1 mostra alguns exemplos que evidenciam a ocorrência destas representações envolvendo interação social, memória espacial e processos de aprendizagem em peixes. Os relatos dos artigos citados são difíceis de interpretação sem recorrer a processos relacionados à senciência.

\section{TABELA 1 - RELATOS CIENTÍFICOS INDICADORES DE REPRESENTAÇÕES MENTAIS DECLARATIVAS EM PEIXES.}

\begin{tabular}{|c|c|c|}
\hline $\begin{array}{l}\text { CARACTERÍSTICAS } \\
\text { COGNITIVAS }\end{array}$ & ESPÉCIES & AUTORES \\
\hline $\begin{array}{l}\text { Uso de informação em interações sociais adquiridas a } \\
\text { partir de observação de coespecíficos. }\end{array}$ & $\begin{array}{l}\text { Guppy (Poecilia reticulata) } \\
\text { Peixe combatente (Betta spendens) } \\
\text { Truta arco-íris (Oncorhynchus mykiss) }\end{array}$ & $\begin{array}{l}\text { DUGATKIN e GODIN (1992) } \\
\text { OLIVEIRA et al. (1998); } \\
\text { MCGREGOR et al. (2001) } \\
\text { JOHNSON e ÅKERMAN (1998) }\end{array}$ \\
\hline $\begin{array}{l}\text { Reconhecimento individual com alteração do comporta- } \\
\text { mento (estratégia de luta, mudança de cor). }\end{array}$ & $\begin{array}{l}\text { Truta arco-íris (Oncorhynchus mykiss) } \\
\text { Salmão do Atlântico (Salmo salar) }\end{array}$ & $\begin{array}{l}\text { JOHNSON (1997) } \\
\text { O'CONNOR et al. (2000) }\end{array}$ \\
\hline $\begin{array}{l}\text { Memória espacial complexa e flexível, com recurso a } \\
\text { diversas estratégias adaptativas incluindo a formação } \\
\text { de mapas cognitivos. }\end{array}$ & $\begin{array}{l}\text { Peixe-capim (Bathygobius soporator) } \\
\text { Peixe-dourado (Carassius auratus) } \\
\text { Salmão do Atlântico (Salmo salar) }\end{array}$ & $\begin{array}{l}\text { ARONSON (1971) } \\
\text { LÓPEZ et al. (2000); } \\
\text { SALAS et al.(1996) } \\
\text { BRAITHWAITE et al. (1996) }\end{array}$ \\
\hline $\begin{array}{l}\text { Associação de estímulos distintos, comportamento de } \\
\text { antecipação e predição das conseqüências dos atos. }\end{array}$ & $\begin{array}{l}\text { Peixe paraíso (Macropodus opercu- } \\
\text { laris) }\end{array}$ & TOPÁL e CSÁNYI (1999) \\
\hline Associação de eventos ao tempo e lugar. & Inanga (Galaxias maculatus) & REEBS (1999) \\
\hline Percepção de lugares perigosos (antecipação). & Esgana-gata (Gasterosteus aculeatus) & $\begin{array}{l}\text { HUNTINGFORD e WRIGHT } \\
\text { (1989) }\end{array}$ \\
\hline Reconhecimento de estilos musicais & Carpas (Cyprinus carpio) & CHASE (2001) \\
\hline
\end{tabular}

FONTE: Adaptado de GALHARDO e OLIVEIRA, 2006. 
Algumas habilidades cognitivas relatadas em peixes apresentam-se particularmente fortes em relação à presença de consciência nestes animais. Os peixes podem extrair informações de eventos externos, nos quais não estão envolvidos, via aprendizado observacional, com subseqüente uso da informação para dirigir comportamento futuro em caminhos alternativos e flexíveis, ou seja, os peixes utilizam habilidades cognitivas (CHANDROO et al., 2004).

\section{APRENDIZADO VIA OBSERVAÇÃO E VIA AUDIÇÃO}

O comportamento dos peixes adquirindo informação via aprendizado observacional pode ser explicado pela hipótese de que os peixes fazem predições do resultado de um ato a partir de observação prévia. Isto sugere que os peixes podem usar representações declarativas sob essas circunstâncias. $\mathrm{O}$ aprendizado observacional mediado pela visualização é um fenômeno complexo, se estende do aprendizado sobre estímulos e objetos no ambiente a respostas comportamentais de animais da mesma espécie, podendo estar envolvido em alguma forma de raciocínio consciente simples (HEYES, 1993).

O'CONNOR et al. (2000) sugeriram que algumas espécies agressivas de peixes de produção podem comunicar-se entre si durante disputas. Em confrontos entre salmão do Atlântico (Salmo salar), por exemplo, um peixe pode repentinamente mudar a cor do seu corpo, o que indica submissão a um oponente. O escurecimento da cor do corpo causa a redução da agressividade do vencedor do combate sobre o peixe escurecido, evitando o prolongamento da luta (O'CONNOR et al., 2000). Durante o contexto de luta, o escurecimento corporal não é considerado um indicador geral de estresse (O'CONNOR et al., 1999).

No estudo realizado por CHASE (2001), verificouse que a carpa comum (Cyprinus carpio) pode discriminar diferentes estilos musicais (blues e música clássica). Os experimentos realizados mostraram que esses animais são capazes de reconhecer um estímulo auditivo complexo e, portanto, demonstrar respostas discriminatórias sofisticadas em relação às suas capacidades auditivas.

A habilidade cognitiva de reconhecimento e realização de certo comportamento que evitará uma situação aversiva é evidência de que o comportamento exibido não seja uma manifestação automática inconsciente (YUE et al., 2004).

\section{SIMILARIDADES NEURONAIS}

Os mecanismos neuronais das características cognitivas de interação social, memória espacial e aprendizagem sugerem similaridades funcionais e de grau de especialização entre peixes e tetrápodos. O telencéfalo dos peixes, quando destruído, resulta nas mesmas perturbações funcionais de formação de mapas cognitivos e de modos de aprendizagem, quando comparado às lesões causadas ao hipocampo dos demais vertebrados (SALAS et al., 1996). Os aprendizados espacial e associativo em peixes-dourados (Carassius auratus) são afetados pela ablação do telencéfalo, de maneira similar à lesão do hipocampo em mamíferos e aves. Isto é, a destruição do telencéfalo dos peixes resulta em perturbações da orientação espacial, em particular da formação de mapas cognitivos (LOPEZ et al., 2000). Novamente, isto sugere que os peixes obtêm representações declarativas.

\section{SISTEMAS LÍMBICO E DOPAMINÉRGICO DE PEIXES}

O sistema límbico é um conjunto de estruturas cerebrais que têm algumas funções em emoções, memória e aprendizado. As emoções envolvem um circuito cerebral primitivo que foi conservado durante a evolução (LeDOUX, 2000, BARRETT et al., 2007). O sistema límbico de tetrápodos consiste de hipocampo, amídala, hipotálamo, habenula e corpos mamilares, enquanto que o sistema límbico de peixes é constituído de telencéfalo, hipotálamo, habenula e corpos mamilares (CHANDROO et al., 2004).

A amídala dos tetrápodos, assim como os corpos mamilares e a habenula, contribuem ao processo olfativo, além de ter influência sobre o despertar e as emoções, como o medo (CHANDROO et al., 2004). Nos peixes existe um complexo amidalar que está localizado no telencéfalo. Lesões provocadas na região do complexo amidalar produzem mudanças na agressividade, semelhantes às observadas quando se provocam lesões amidalares em tetrápodos (BRADFORD, 1995). O hipotálamo de tetrápodos está relacionado com a integração e controle de uma variedade de funções autônomas, comportamento sexual e emoções. O hipotálamo dos peixes está envolvido no comportamento sexual e outros comportamentos sociais, sendo também responsável pela integração de sinais ambientais, incluindo os sinais internos surgidos no telencéfalo, originando o aprendizado emocional (FOX et al., 1997). 
Em conjunto com o sistema límbico, sistemas neuronais dopaminérgicos específicos estão envolvidos em alguns tipos de processos afetivos e motivacionais. Os neurônios dopaminérgicos mesolímbicos projetam-se para dentro do sistema límbico, mediando os mecanismos de aprendizado e recompensa em tetrápodos. Estes neurônios têm sido relacionados ao controle e à expressão do comportamento resultantes da emoção. Existem dados que indicam que a inervação dopaminérgica do telencéfalo de peixes media os estados motivacionais e o comportamento de maneiras similares aos tetrápodos (CHANDROO et al., 2004). Esta hipótese é baseada em estudos farmacológicos que investigam os efeitos da substância $P$ no comportamento de vertebrados. Juntamente à mediação da dor, o neuropeptídeo substância $P$ está envolvido nos processos de aprendizado, memória e recompensa em mamíferos, e seus efeitos podem ser mediados através do sistema dopaminérgico (MATTIOLI et al., 1997). Nos peixes, assim como em mamíferos, os corpos celulares dopaminérgicos estão localizados em regiões similares do cérebro às que são encontrados os terminais de substância P. Nos peixes, há evidências de que os agonistas dopaminérgicos influenciam o comportamento e estão relacionados com a regulação mesolímbica da agressão e do medo (SNEDDON, 2002; SNEDDON et al., 2003).

Portanto, as características anatômicas, fisiológicas e comportamentais dos peixes demonstram que eles apresentam estruturas dopaminérgicas e límbicas com funcionamento homólogo aos tetrápodos. A presença destes sistemas neuronais em peixes, com um sistema semelhante de memória declarativa, sugere que os peixes modernos e os tetrápodos herdaram estes traços de um ancestral comum durante a evolução dos vertebrados. Alguns destes traços devem ter sido conservados sem extremas modificações, sendo que o conhecimento atual sugere que as emoções envolvem circuitos cerebrais relativamente primitivos que foram conservados durante a evolução (CHANDROO et al., 2004).

As semelhanças encontradas entre o funcionamento dos sistemas límbico e dopaminérgico dos peixes e dos tetrápodos indicam que os peixes estão aptos a sentir emoções e possuem capacidade de memória e de aprendizado.

\section{ESTADOS AFETIVOS MOTIVACIONAIS}

Os estados afetivos motivacionais incluem um vasto grupo de experiências conscientes como dor, medo, fome, sede e prazer. Eles são reconhecidos como produtos adaptativos da seleção natural e são importantes na formação de certos tipos de compor- tamento (FRASER e DUNCAN, 1998).

O medo é uma emoção que resulta em alterações fisiológicas e comportamentais imediatas à percepção de um estímulo perigoso (GALHARDO e OLIVEIRA, 2006), e se constitui num motivador poderoso para evitar ameaças percebidas (JONES, 1997). Os indicadores de medo que têm sido referenciados para peixes incluem o aumento da taxa respiratória, a produção de feromônios de alarme e as reações comportamentais de aversão, nomeadamente a fuga rápida e o distanciamento (CHANDROO et al., 2004). Usando paradigmas de condicionamento operacional e clássico, YUE et al. (2004) demonstraram como trutas arco-íris (Oncorhynchus mykiss) aprendem através de uma associação de estímulos, a evitar um evento percebido como perigoso, sendo capazes de obter um aprendizado envolvendo memória de longa duração (mais de sete dias). $O$ tipo de resposta comportamental exibida (fuga) mostrou-se flexível e adaptativo, pois o seu tempo de latência variou com a exposição ao estímulo, sendo menor na presença do evento adverso (estímulo não condicionado) que em relação ao estímulo condicionado (que originalmente antecedia e sinalizava a ocorrência do evento adverso). Isto depende de mecanismos cognitivos e de regiões cerebrais homólogas aos dos tetrápodos (YUE et al., 2004).

As alterações comportamentais verificadas constituem mecanismos adaptativos que visam, em geral, reduzir ou eliminar a exposição a agentes estressores. Quando possível, o comportamento mais imediato é a fuga ou imobilização. Se o contexto ambiental não permite a fuga, são observadas alterações significativas do comportamento dos peixes, tais como mudanças no ritmo e padrão natatório, redução ou alteração do comportamento anti-predatório, mudança do comportamento alimentar, aumento da procura de abrigo, redução ou aumento de comportamentos agonísticos e alterações da capacidade de aprendizagem. SNEDDON et al. (2003) concluíram que na resposta à dor os peixes sentem-se emocionalmente estressados e mostram respostas extremas semelhantes àquelas vistas em outros vertebrados, como o aumento da taxa respiratória e do tempo para retornar a alimentar-se. Adicionalmente, segundo TAVOLGA (1982), os bagres marinhos (Arius felis) utilizam sons para comunicar o medo quando redes são mergulhadas em seus tanques ou quando são manejados.

Em algumas espécies de peixes, a resposta ao medo e ansiedade pode ser demonstrada pela exsudação de feromônios através da pele (SMITH, 1992). Adicionalmente, a indução de reações de alarme por peixes pode ser transferida para outro estímulo que inicialmente não provoque o alarme. Reações de alarme e comportamentos defensivos 
Bem-estar de peixes e a questão da senciência

anti-predatórios foram provocados em paulistinhas (Brachydanio rerio) por meio de feromônio exsudado pela pele ferida de peixes coespecíficos (HALL e SUBOSKI, 1995), fato encontrado quando um estímulo olfatório inócuo (morfolina) apresentado aos peixes juntamente com uma substância de alarme, eliciou a transformação da morfolina em um eliciador de alarme pelos peixes. Os achados iniciais de transmissão social de reconhecimento predatório em paulistinhas foram confirmados mais detalhadamente quando os peixes treinados por condicionamento clássico à reação de alarme à luz e à morfolina foram misturados com peixes não condicionados e foram testados com luz e com morfolina isoladamente. Todos os peixes do grupo treinado dispararam alarme. Os peixes que não haviam sido treinados foram separados e testados novamente com luz e com morfolina. Todos eles exibiram reação de alarme e, portanto, aprenderam pela interação social a reconhecer o perigo. Ou seja, os autores demonstraram que a indução de comportamento de medo pode ser transferida através de processos de aprendizado associativos, do feromônio para o estímulo inócuo visual ou olfatório e pela interação social.

Ainda, a resposta ao medo por feromônio diminui quando os peixes são tratados com medicamentos ansiolíticos que se ligam aos receptores específicos de benzodiazepina no cérebro (REHNBERG et al., 1989). WISENDEN e SMITH (1998) mostraram que os peixes ajustam a síntese de feromônio de alarme, baseando-se no nível de risco percebido no ambiente. Estes achados sugerem que os estados de medo e ansiedade nos peixes são mediados por sistemas límbicos neuronais de forma similar aos mamíferos.

\section{NOCICEPÇÃO E PERCEPÇÃO DA DOR}

A nocicepção é definida como a capacidade de deteç̧ão e reação a um estímulo nocivo que possa comprometer a integridade física do animal (KAVALIERS, 1988). Já a dor pode ser definida como experiência sensorial e emocional desagradável associada a dano tecidual (ROSE, 2002). Em outras palavras, a nocicepção indica os aspectos sensóriomotores sem caracterizar as reações subjetivas e o sofrimento (MATHER, 2001).

A questão da dor tem um enorme significado para o bem-estar animal, sendo citada no contexto das cinco liberdades: nutricional, sanitária, ambiental, psicológica e comportamental (MOLENTO, 2005). A habilidade de sentir dor é geralmente associada às capacidades de animais sencientes, sendo uma causa importante de baixo grau de bem-estar. $O$ sistema relacionado à consciência da dor inclui uma análise cerebral complexa (BROOM, 2007). Recentemente, a ocorrência de dor em peixes tem sido debatida mais intensamente (ROSE, 2002; SNEDDON, 2002; SNEDDON et al. 2003; JACKSON, 2003; CHANDROO et al., 2004; BRAITHWAITE e HUNTINGFORD, 2004; BRAITHWAITE $\mathrm{C}$ BOULCOTT, 2007; BROOM, 2007), sendo possível perceber que ainda há muito a se pesquisar sobre a questão de como os peixes são capazes de perceber a dor e qual a intensidade dessa percepção.

O nervo trigêmio, quinto par de nervos cranianos, transmite a maioria das informações sensoriais da cabeça dos vertebrados para o cérebro (SNEDDON et al., 2003). A dor é transmitida por duas classes de nervos nociceptivos: (i) fibras delta-A, que são de condução rápida, pequenas e mielinizadas, e são associadas com a dor primária (particularmente em relação ao estímulo nocivo térmico) e; (ii) fibras $\mathrm{C}$, que são condutoras mais lentas, menores e nãomielinizadas, e são associadas com a dor secundária, sensação desagradável mais longa relacionada ao dano tecidual (BRAITHWAITE e BOULCOTT, 2007). Ambas as fibras são encontradas no nervo trigêmio da truta arco-íris (Oncorhyncus mykiss). Registros eletrofisiológicos feitos nos corpos celulares do gânglio trigeminal das trutas mostraram que os receptores isolados na cabeça e na face foram hábeis para detectar estímulos nocivos de pressão mecânica, temperatura e estímulo químico, confirmando a presença e a atividade dos nociceptores (SNEDDON et al., 2003). Ainda, foi identificado um conjunto de comportamentos em resposta à estimulação nociva, indicadores de processamento cerebral. Este trabalho de SNEDDON et al. (2003) sugere que o peixe experimenta a dor de maneira similar aos mamíferos.

Adicionalmente, de acordo com BRAITHWAITE e BOULCOTT (2007), a verificação de que os peixes apresentam os mesmos tipos de nociceptores de outros vertebrados, que os impulsos nervosos são conduzidos ao telencéfalo e que a motivação e o comportamento são afetados por estímulos nocivos, reforça a evidência de que os peixes podem sentir e reagir conscientemente a esses estímulos.

Desta forma, as evidências científicas atuais apontam no sentido do reconhecimento de peixes como seres sencientes. Recomenda-se a consideração de peixes como seres sencientes, embora a demonstração cabal de senciência nesses, como em quaisquer outros animais, seja elusiva. Tal recomendação encontra bases em uma abordagem que combine ciência e ética, como proposta por VOLPATO et al. (2007). Também por uma análise mais pragmática parece a recomendação mais sensata, pois se trata de pesar todas as evidências científicas de senciência em peixes discutidas acima 
e contrastar com a realidade de que não existe um único relato científico que evidencie a ausência de sentimentos nestes animais. Finalmente, o reconhecimento da senciência em peixes traz consigo a demanda de reflexão sobre práticas comumente empregadas em piscicultura.

\section{BEM-ESTAR DE PEIXES E A PISCICULTURA}

Para garantir-se bem-estar de peixes de produção, três áreas de entendimento precisam ser desenvolvidas: (1) a compreensão dos processos biológicos envolvidos, (2) os aspectos morais da sociedade e (3) as estratégias para o balanceamento de conflitos de interesse: peixes, ambiente e ser humano (HUNTINGFORD et al., 2006).

TURNBULL e KADRI (2007) propuseram um esquema (FIGURA 1) para sumarizar as necessidades de adaptação que os animais enfrentam em termos de produção pecuária: (A) os peixes apresentam uma gama de possibilidades de adaptação que cumprem sua função em seu ambiente natural, (B) um ambiente artificial, imposto pelo ser humano para fins de produção ou outros, que oferece desafios aos quais os peixes apresentam capacidade de adaptação a partir de suas capacidades naturais, e (C) um ambiente artificial, imposto pelos seres humanos, que oferece desafios para os quais os peixes não apresentam capacidade de adaptação com sucesso do ponto de vista do seu bem-estar. O aumento na densidade de lotação e a conseqüente diminuição da qualidade da água são exemplos de fatores que aumentam o risco de comprometimento do processo de adaptação e queda no grau de bem-estar, representados pela seta $D$. A seta $E$ representa fatores que podem contribuir para um aumento no grau de bem-estar por meio da facilitação dos processos de adaptação ao meio, como por exemplo, planejamento de contingência, treinamento de pessoal e enriquecimento ambiental.

\section{FIGURA 1 - MODELO DE APRESENTAÇÃO DE INFORMAÇÕES RELATIVAS AO BEM-ESTAR DE PEIXES DE PRODUÇÃO. O CÍRCULO DA ESQUERDA REPRESENTA A ADAPTAÇÃO DO PEIXE EM AMBIENTE NATURAL E O CÍRCULO DA DIREITA O SISTEMA DE PRODUÇÃO. AS CINCO ÁREAS A, B, C, D E E PODEM SER PREENCHIDAS POR INFORMAÇÕES SOBRE A NATUREZA DO PEIXE, SUAS ADAPTAÇÕES E DESCRIÇÕES DO SISTEMA DE PRODUÇÃO.}

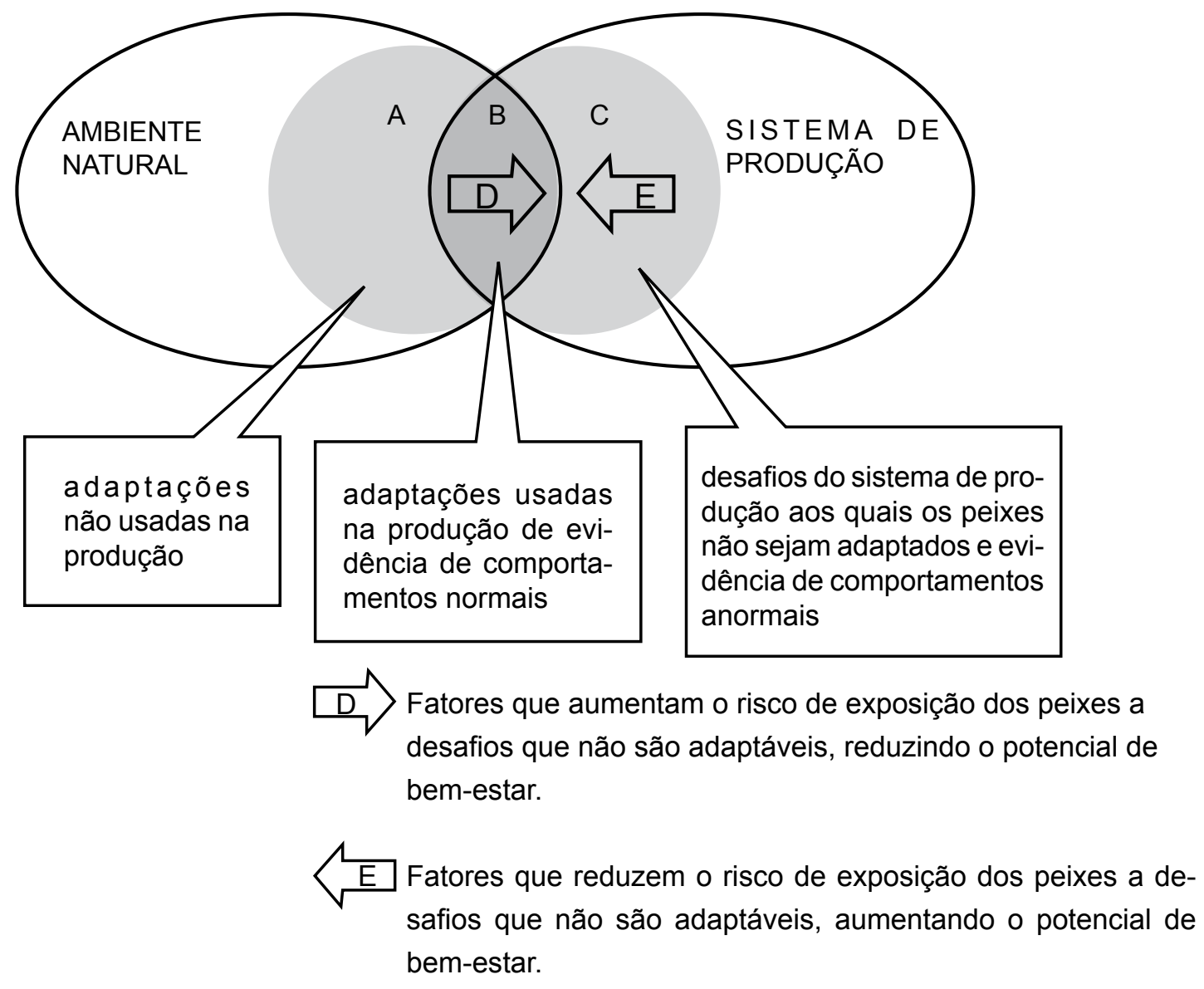

(adaptado de TURNBULL e KADRI, 2007). 
Para a construção do contexto global dos sistemas produtivos em termos de bem-estar, é necessária a discussão de situações mais pontuais. A alimentação, o manejo, a qualidade da água, densidade de lotação, o transporte e o abate são os principais pontos críticos da produção de peixes, podendo interferir no seu grau de bem-estar. Estes pontos críticos são discutidos mais detalhadamente por PEDRAZZANI et al. (2007).

\section{ESTRATÉGIAS DE MELHORIA: TESTES PREFERENCIAIS}

Os testes preferenciais aplicados aos peixes poderiam fornecer informações úteis sobre as condições mais agradáveis para os indivíduos em relação ao espaço, cor e intensidade de luz, horário e quantidade de alimentação, necessidade de sombreamento, profundidade da coluna de água e seu fluxo (VOLPATO, 2007; VOLPATO et al., 2007). Isso facilitaria o emprego do enriquecimento ambiental, que é um conjunto de técnicas que modificam o ambiente, resultando em uma melhora na qualidade de vida dos animais, ao satisfazer as suas necessidades comportamentais, podendo diminuir o estresse e meIhorar o bem-estar (BOERE, 2001). Face às características ecológicas de algumas espécies, certo grau de complexidade ambiental poderia ser importante (FSBI, 2002). Conclui-se que os locais de abrigo, o uso de diferentes substratos e correntes de água, formas estratégicas de distribuição de alimento, entre outros manejos, poderiam ter um papel relevante nas preferências e opções dos peixes, incrementando assim o seu bem-estar.

\section{CONCLUSÃO}

Os peixes teleósteos, apesar de apresentarem diferenças em alguns aspectos da estrutura cerebral se comparados aos tetrápodos, possuem similaridades funcionais e habilidades cognitivas sugestivas de senciência. Os dados anatômicos, fisiológicos, comportamentais, evolutivos e farmacológicos evidenciam que os peixes experimentam sentimentos como dor e medo de maneira similar aos demais vertebrados. Isso sugere que os peixes têm a capacidade de sofrer e que, portanto, estes animais devem ser incluídos nas considerações éticas acerca de não se causar sofrimento.

\section{REFERÊNCIAS}

APPLEBY, M. What Should We Do About Animal Welfare? Oxford: Blackwell Science, 192 pp.,1999.

ARONSON, L.R. Further studies on orientation and jumping behaviour in the Gobiid fish Bathygobius soporator. Annals of the New York Academy of Science, v. 188, p. 378-392, 1971.

BARRETT, L.F.; MESQUITA, B.; OCHSNER, K.N.; GROSS, J.J. The Experience of Emotion. Annual Review of Psychology, v. 58, p. 373-403, 2007.

BEKOFF, M. Animal emotions and animal sentience and why they matter: blending "science sense" with common sense, compassion and heart. In: TURNER, J.; D'SILVA, J. Animals, ethics, and trade: The Challenge of Animal Sentience. Earthscan Publishing: London, 2006. p. 27-40.

BENSON, J.; ROLLIN, B.E. The Well-Being of Farm Animals: Challenges and Solutions. Blackwell Publishing: Ames, lowa, 388 pp., 2004.

BOERE, V. Enriquecimento ambiental para primatas neotropicais em cativeiro. Ciência Rural, v. 31, n. 3, p. 543-551, 2001.

BRADFORD, Jr; M.R. Comparative aspects of forebrain organization in the ray-finned fishes: touchstones or not? Brain, Behavior and Evolution, v. 46, p. 259-274, 1995.

BRAITHWAITE, V.A.; ARMSTRONG, J.D.; McAdAM, H.M.; HUNTINGFORD, F.A. Can juvenile Atlantic salmon use multiple cue systems in spatial learning? Animal Behaviour, v. 51, p. 1409-1415, 1996.

BRAITHWAITE, V.A.; HUNTINGFORD, F.A. Fish and welfare: do fish have the capacity for pain perception and suffering? Animal Welfare, v.13, p. 587-592, 2004.

BRAITHWAITE, V.A; BOULCOTT, P. Pain perception, aversion and fear in fish. Disease of Aquatic Organisms, v.75, p.131-138, 2007.

BRANSON, E. Fish Welfare. Blackwell Publishing. 352 pp., 2007. 
BROOM, D.; MOLENTO, C. Bem-Estar Animal: Conceito e Questões Relacionadas - Revisão. Archives of Veterinary Science, v. 9, n. 2, p. 1-11, 2004.

BROOM, D.M. Cognitive ability and sentience: Which aquatic animals should be protected? Disease of Aquatic Organisms, v. 75, p. 99-108, 2007.

BSHARY, R.; WICKLER, W.; FRICKE, H. Fish cognition: a primate eye view. Animal Cognition, v. 5 , p. 1-13, 2002.

CHANDROO, K.P.; DUNCAN, I.H.; MOCCIA, R.D. Can fish suffer? Perspectives on sentience, pain, fear and stress. Applied Animal Behavior, v. 86, p. 225-250, 2004.

CHASE, A. R. Music discrimination by carp (Cyprinus carpio). Animal learning and behaviour, v. 29, n. 4, p. 336-353, 2001.

DOLAN, K. Ethics, Animals and Science. lowa State University Press: Ames, 302 pp., 1999.

DUGATKIN, L.A.; GODIN, J.G.J. Reversal of female mate choice by copying in the guppy Poecilia reticulata. Proceedings Royal Society of London, Biological Sciences, v. 249, p. 179-184, 1992.

DUNCAN, I.J. Science-based assessment of animal welfare: farm animals. Revue Scientifique et Technique OIE, v. 24, n. 2, p. 483-492, 2005.

DUNCAN, I.J.; PETHERICK, J.P. The implications of cognitive processes for animal welfare. Journal of Animal Science, v. 69, n. 12, p. 5017-5022, 1991.

DUNCAN, I.J.H. Animal welfare defined in terms of feelings. Acta Agriculturæ Scandinavica Section A, Animal Science, v. 2, p. 29-35,1996.

EDELMAN, G.M.; TONONI, G. Universe of Consciousness. Basic Books, United States of America, 274 pp., 2000.

ERICKSON, H.S. Information Resources on Fish Welfare: 1970-2003. AWIC Resource Series No. 20. U.S. Dept. of Agriculture, National Agricultural Library, Animal Welfare Information Center. Beltsville, MD., 436 pp., 2003.
FOX, H.E.; WHITE, S.A.; KAO, M.H.F.; FERNALD, R.D. Stress and dominance in a social fish. Journal of Neuroscience, v. 17, p. 6463-6469, 1997.

FRASER, A. F.; BROOM, D. M. Farm Animal Behaviour and Welfare. Wallingford: Cab International. $3^{\text {a Ed., }} 448$ pp., 1996.

FRASER, D.; DUNCAN, I.J.H. 'Pleasures', 'pains' and animal welfare: toward a natural history of affect. Animal Welfare, v. 7, p. 383-396, 1998.

FSBI. Fish Welfare (Briefing Paper 2). Fisheries Society of the British Isles. Cambrigde: Granta Informations Systems, 2002. Disponível em www.le.ac. uk/biology//briefing. Acesso em: 20 jan. 2006.

GALHARDO, L.; OLIVEIRA, R. Bem-estar Animal: um conceito legítimo para peixes? Revista de Etologia, $v$. 8, n. 01, p.51-61, 2006.

GRIFFIN, D. R. Animal Minds. University of Chicago Press: Chicago, IL, 320 pp, 1992.

GRIFFIN, D. R.; SPECK, G.D. New evidence of animal consciousness. Animal Cognition, v. 7, p. 5-18, 2004.

HALL, D.; SUBOSKI, M.D. Visual and olfactory stimuli in learned release of alarm reactions by zebra danio fish (Brachydanio rerio). Neurobiology of Learning and Memory, v. 63, n. 3, p. 229-40, 1995.

HEYES, C.M. Imitation, culture and tradition, Animal Behaviour, v. 46, p. 999-1010, 1993.

HUNTINGFORD, F.A.; ADAMS, C.; BRAITHWAITE, V.A.; KADRI, S.; POTTINGER, T.G.; SANDØE, P.; TURNBULL, J.F. Review paper: Current issues in fish welfare. Journal of Fish Biology, v. 68, p. 332-372, 2006.

HUNTINGFORD, F.A.; WRIGHT, P.J. How sticklebacks learn to avoid dangerous feeding patches. Behavioural Processes, v. 19, p. 181-189, 1989.

JACKSON, C. Laboratory fish: impacts of pain and stress on well-being. Contemp. Top Laboratory Animal Science, v. 42, p. 62-73, 2003.

JOHNSON, J.I. Individual recognition affects aggression and dominance relations in rainbow trout, Oncorhynchus mykiss. Ethology, v. 103, p. 267-282, 1997. 
Bem-estar de peixes e a questão da senciência

JOHNSON, J.I.; ÅKERMAN, A. Watch and learn: preview of the fighting ability of opponents alters contest behaviour in rainbow trout. Animal Behaviour, v. 56, p. 771-776, 1998.

JONES, B.R. Fear and distress, p. 75-87. In: APPLEBY, M.C., HUGHES, B.O. Animal Welfare. CAB International: Wallingford, 316 pp., 1997.

KAVALIERS, M. Evolutionary and comparative aspects of nociception. Brain Research Bulletin, v. 21, p. 923-931, 1988.

KIRKWOOD, J.K. The distribution of the capacity for sentience in the animal kingdom, In: TURNER, J. ; D'SILVA, J. Animals, ethics, and trade: The Challenge of Animal Sentience. Earthscan Publishing: London, 2006, p.12-25.

LeDOUX, J. E. Emotion circuits in the brain. Annual Review of Neurosciences, v. 23, p. 155-184, 2000.

LÓPEZ, J.C.; BINGMAN, V.P.; RODRIGUEZ, F.; GÓMEZ, Y.; SALAS, C. Dissociation of place and cue learning by telencephalic ablation in goldfish. Behavioral Neuroscience, v. 114, p. 687-699, 2000.

LYMBERY, P. In too deep: The welfare of intensively farmed fish. CIWF - Compassion In World Farming: Hampshire, 56 pp., 2002.

MATHER, J.A. Animal suffering: An invertebrate perspective. Journal of Applied Animal Welfare Science, v. 4, p. 151-156, 2001

MATTIOLI, R.; SANTANGELO, E.M.; COSTA, A.C.C.; VASCONCELOS, L. Substance $P$ facilitates memory in goldfish in an appetitive motivated learning task. Behavioural Brain Research, v. 85, p. 117-120, 1997.

McGREGOR, P.K.; PEAK, T.M.; LAMPE, H.M. Fighting fish Betta splendens extracts relative information from apparent interactions: what happens when what you see is not what you get. Animal Behaviour, v. 62, p. 1059-1065, 2001.

MOLENTO, C.F.M. Bem-estar e produção animal: aspectos econômicos - Revisão. Archives of Veterinary Science, v. 10, n. 1, p. 1-11, 2005.
O'CONNOR, K.I., METCALFE, N.B., TAYLOR, A.C., 1999. Does darkening signal submission in territorial contests between juvenile Atlantic salmon, Salmo salar? Animal Behaviour, v. 58, p.1269-1276, 1999.

O'CONNOR, K.I., METCALFE, N.B., TAYLOR, A.C. Familiarity influences body darkening in territorial disputes between juvenile salmon. Animal Behaviour, v. 59, p. 1095-1101, 2000.

OLIVEIRA, R.F.; McGREGOR, P.K.; LATRUFFE, C. Know thine enemy: Fighting fish gather information from observing conspecific interactions. Proceedings ... Royal Society of London, Biological Sciences, v. 265, p. 1045-1049, 1998.

PEDRAZZANI, A.S., MOLENTO, C. F. M., CARNEIRO, P.C.F., FERNANDES-DE-CASTILHO, M. Senciência e bem-estar de peixes: uma visão de futuro do mercado consumidor. Panorama da Aqüicultura, v. 102, p. 24-29, 2007.

PRADA, I. A alma dos animais. Editora Mantiqueira: Campos do Jordão, 63pp., 2000.

REEBS, S.G. Time-place learning based on food but not on predation risk in a fish the inanga (Galaxias maculates). Ethology, v. 105, p. 361-371, 1999.

REHNBERG, B.G.; BATES, E.H.; SMITH, R.J.F.; SLOLEY, B.D.; RICHARDSON, J.S. Brain benzodiazepine receptors in fathead minnows and the behavioral response to alarm pheromone. Pharmacology, Biochemistry, Behavior, v. 33, p. 435-442, 1989.

ROLLIN, B.E. Farm Animal Welfare: School, Bioethics and Research Issues. Iwoa State Press, 168pp., 1995.

ROSE, J.D. The neurobehavioral nature of fishes and the question of awareness and pain. Reviews in Fisheries Science, v. 10, p. 1-38, 2002.

SALAS, C.; BROGLIO, C.; RODRIGUEZ, F.; LÓPEZ, J.C.; PORTAVELLA, M.; TORRES, B. Telencephalic ablation in goldfish impairs performance in a 'spatial constancy' problem but not in a cued one. Behavioural Brain Research, v. 79, p. 193-200, 1996.

SHETTLEWORTH, S. J. Animal cognition and animal behaviour. Animal Behaviour, v. 61, p. 277-286, 2001. 
SMITH, R.J.F. Alarm signals in fishes. Reviews in Fish Biology and Fisheries, v. 2, p. 33-63, 1992.

SNEDDON, L.U. Anatomical and electrophysiological analysis of the trigeminal nerve in a teleost fish, Oncorhynchus mykiss. Neuroscience Letters, v. 319, p. 167-171, 2002.

SNEDDON. L.U.; BRAITHWAITE V.A. GENTLE M.A. Do fishes have nociceptors? Evidence for the evolution of a vertebrate sensory system. Proceedings of the Royal Society Biological Science, v. 270, p. 1115-1121, 2003.

TAVOLGA, W.N. Auditory acuity in the sea catfish (Arius felis). Journal of Experimental Biology, v. 96, p. $367-376,1982$.

TOPÁL, J.; CSÁNYI, V. Interactive learning in the paradise fish (Macopodus opercularis): an ethological interpretation of the second-order conditioning paradigm. Animal Cognition, v. 2, p. 197-206, 1999.

TURNBULL, J.F.; KADRI, S. Safeguarding the many guises of farmed fish welfare. Disease of Aquatic Organisms, v. 75, p. 173-182, 2007.

VAARST, V.; RODERICK, S.; LUND, V.; LOCKERETZ, W. Animal Health and Welfare in Organic Agriculture. Cab International: Cambridge, 448 pp., 2004.

VOLPATO, G.L. Considerações metodológicas sobre o teste de preferência na avaliação do bem-estar em peixes. Revista Brasileira de Zootecnia, v. 36, suplemento especial: 53-61, 2007.

VOLPATO, G.L.; GONÇALVES-DE-FREITAS, E.; FERNANDES-DE-CASTILHO, M. Insights into the concept of fish welfare. Disease of Aquatic Organisms, v. 75, p. 165-171, 2007.

WISENDEN, B.D.; SMITH, R.J.F. A re-evaluation of the effect of shoalmate familiarity on the proliferation of alarm substance cells in Ostariophysan fishes. Journal of Fish Biology, v. 53, p. 841-846, 1998.

YUE, S., MOCCIA, R.D., DUNCAN, I.J. Investigating fear in domestic rainbow trout, Oncorhynchus mykiss, using an avoidance learning task. Applied Animal Behaviour Science, v. 87, p. 343-354, 2004.

Recebido para publicação: 